Journal of Engineering and Applied Sciences 14 (1): 170-177, 2019

ISSN: 1816-949X

(C) Medwell Journals, 2019

\title{
Low Cost Arduino Based Smart Energy Monitoring System Using Internet of Things
}

\author{
${ }^{1} \mathrm{R}$. Govindarajan, ${ }^{2} \mathrm{~S}$. Meikandasivam and ${ }^{2} \mathrm{D}$. Vijayakumar \\ ${ }^{1}$ Department of EEE, SCSVMV, Enathur, Kanchipuram, Tamil Nadu, India \\ ${ }^{2}$ School of Electrical Engineering, VIT, Vellore, Tamil Nadu, India
}

\begin{abstract}
The proposed concept promotes smart energy monitoring system using Internet of Things (IoT) techniques. This wireless energy monitoring system can collect the parameters from the digital energy meter through the internet communication. In addition with the system can generate real time power consumption report in online. So that, the user can save the electricity and get the awareness about the power utilization. Now a days, the electricity board using traditional analog meter as well as digital meter even though the reading was not suitable for longer operating purposes as it spends much human and material resource. It brings additional problems in calculation of readings and billing manually. It's a hard task to maintain the power as per the growing requirements. If the consumer is not available, the billing process will be pending and human operator needs to revisit every consumer's house and the operator needs to go to their houses to disconnect the power supply if bill not paid. So, the problem which arises in the billing system can become inaccurate and inefficient. The availability of wireless communication media has made the exchange of information fast, secured and accurate, a system called smart energy meter that collects the energy consumption from residential as well as corporate zones and send it directly to the central server where processing is done on that data for preparation of bills to overcome the drawback of manual billing system.
\end{abstract}

Key words: Smart energy meter, IoT techniques, embedded design, requirement, communication, energy consumption

\section{INTRODUCTION}

Now a days, electricity has become a basic need to humans. The consumption of power has increased a lot compared to the past years. The wastage electricity has also become a problem these days and there is no control over the loss due to the unwanted power utilization. In our concept, we planned to implement a low cost smart energy monitoring system using IoT techniques to measure the consumption of the power by the individual and provide awareness to the consumers to know about their power utilization.

A smart meter is usually two way communication based electrical meter that records and measures the electrical parameters in intervals of an hour or less and communicates that information at least daily back to the utility for monitoring and some other official purposes. Smart meters enable two-way communication between the meter and the server system. Also, the smart meters can gather the data by wireless using GSM/ZigBee/IoT techniques. Smart energy meter is software based, digitalized and computerized devices which gives real time parameter in online.
Here, the electrical parameters can be transmitted to distributors/utilities through advanced wireless techniques. Using a smart energy meters, we can sit on our home and simply monitor the electrical parameters from our electricity meter and let the consumer know about the power utilization this put more control on your hands on how you spend your energy at home. But the traditional electricity meters are normally fixed on a wall and the only time you can realize how much energy you have utilized when assessor will calculate the bill.

Literature review: Sensing, monitoring and actuating systems are expected to play a key role in reducing buildings overall energy consumption. Leveraging sensor systems to support energy efficiency in buildings poses novel research challenges in monitoring space usage, controlling devices, interfacing with smart energy meters and communicating with the energy grid. In the attempt of reducing electricity consumption in buildings, identifying individual sources of energy consumption is key to generate energy awareness and improve efficiency of available energy resources usage. Previous research studied several non-intrusive load monitoring techniques

Corresponding Author: R. Govindarajan, Department of EEE, SCSVMV, Enathur, Kanchipuram, Tamil Nadu, India 
to classify appliances, however, the literature lacks of an comprehensive system that can be easily installed in existing buildings to empower users profiling, benchmarking and recognizing loads in real-time. This has been a major reason holding back the practice adoption of load monitoring techniques (Ruzzelli et al., 2010).

In this study, we present RECAP: Recognition of Electrical Appliances and Profiling in real-time. RECAP uses a single wireless energy monitoring sensor easily clipped to the main electrical unit. The energy monitoring unit transmits energy data wirelessly to a local machine for data processing and storage. The RECAP system consists of three parts: guiding the user for profiling electrical appliances within premises and generating a database of unique appliance signatures, using those signatures to train an artificial neural network that is then employed to recognize appliance activities and providing a load descriptor to allow peer appliance benchmarking.

RECAP addresses the need of an integrated and intuitive tool to empower building owners with energy awareness. Enabling real-time appliance recognition is a stepping-stone towards reducing energy consumption and allowing a number of major applications including load-shifting techniques, energy expenditure breakdown per appliance, detection of power hungry and faulty appliances and recognition of occupant activity.

Towards sustainable growth (ICEPE) electrical utilities are suffering from huge losses due to power theft, inadequate usage of energy, unpaid bills and distorted power quality. Many viable solutions are proposed out of which smart energy metering, energy management system and smart home automation are some that seems to be potent enough. These technologies are presently used by developed countries. Under meter data acquisition system of Restructured Accelerated Power Development and Reform Program (R-APDRP) scheme in the year 2009-10, India plans to develop advanced metering infrastructure (Das and Saikia, 2015).

But no effort has been made to develop an economic model for the customers which would play a key role in better acceptance of the scheme. In this study, a smart energy system for the residential customers is developed and a smart switch board which can curtail the need to upgrade to smart appliances to make the system more economic. Further, a virtual instrumentation has been developed which can be operate in any computer to function as in Home Display (IHD) for Energy Management System (EMS).

The demand for energy is increasing due the growth of the population and industrial development. To improve the energy efficiency, consumers should be well informed of their energy consumption. Throughout the past few years, the welfare services have begun to develop new electrical energy meters which are known the smart meters, they are is a efficient and performed interactivity between supplier and consumer (Tariq, 2014). This study presents an approach in the pervasive systems domain, the suppliers would control the energy consumption in given houses by cell phones based on data sent by sensors installed on different corners of houses from the Arduino card based upon ZigBee protocol which will store the information and communicate with consumer via. SMS. For this, programmable Arduino card via. Arduino Software and programmable XBee module by $\mathrm{X}$-CTU Software is used.

The growing demand of energy and the need of finding alternative energy sources to the traditional ones due to the progressive decrease of fossil fuels and an increasing concern towards the environment have led to a revolution in terms of energy production in the last decade. As a consequence, the distributed generation is more and more widely spreading. The network, in this new dimension has to change its management and the energy distribution, so, to achieve and maintain high efficiency requirements (De Capua et al., 2014).

Coming to drop the concept of centralized production, it is immediate to conclude that an efficient distribution of energy must necessarily bring into account the energy footprint of the area because the energy transport should be always as short as possible, to minimize losses and maximize the efficiency of the network. This concept is the core of the smart-grid idea, on which the global scientific community is investing heavily in research, the idea is a power distribution grid, based on the experience in the information and communications technology field which can route the energy through appropriate algorithms that are able to determine the optimal path. Of course, behind all this there must be a network structure capable of acquiring detailed data from widespread production and consumption of energy and make them easily available along with additional information, e.g., the power quality of the energy exchanged. This information is demanded by simple user who wants to personally evaluate the function of the system and also by technical personnel who needs to access to reliable data to perform targeted and efficient interventions. In the present study, the authors propose a smart energy meter for energy management in power grids.

Emerging new technologies like distributed generation, distributed storage and demand-side load management will change the way, we consume and 
produce energy. These techniques enable the possibility to reduce the greenhouse effect and improve grid stability by optimizing energy streams. By smartly applying future energy production, consumption and storage techniques, a more energy-efficient electricity supply chain can be achieved. In this study, a three-step control methodology is proposed to manage the cooperation between these technologies, focused on domestic energy streams (Molderink et al., 2010).

In this approach (global) objectives like peak shaving or forming a virtual power plant can be achieved without harming the comfort of residents. As shown in this research, using good predictions in advance planning and real-time control of domestic appliances, a better matching of demand and supply can be achieved.

Existing system: In existing system for collection of energy consumption data is that the representatives of Tamil Nadu Electricity Board monthly comes and visit every residential, take the snap shot and corporate and manually reads the consumption data from the meter. This collected data is recorded on a piece of paper along with a snap shot of the meter and finally, submitted to the local Tamil Nadu Electricity Board Office. There after the official's read the snap shot and meter readings and then gives it to the local software for bill calculations and generation of bill. We as a consumer then make the payment for the received bill.

Figure 1 shows that the traditional electricity bill is followed as modelled in the block diagram, the conventional energy meter placed in residential or industrial area is checked manually by the $\mathrm{EB}$ member, the data is collected directly and bill is collected manually.

This process is so much hectic process. Man made mistakes can be countless. Human resources wasted and many other problems do occur. We finally thought of building a system that will do the above process automatically (Das and Saikia, 2015). Arduino is attached with our traditional energy meters that will scan the meter

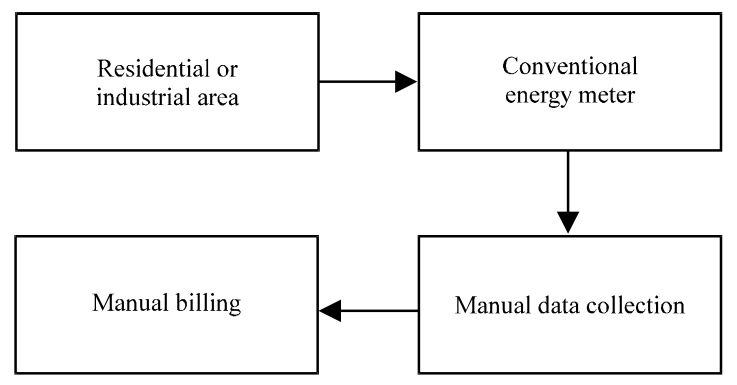

Fig. 1: Conventional energy meter reading after particular period. Wirelessly, these meters reading will transmitted to the centralized server along with their unique meter number.

\section{MATERIALS AND METHODS}

In the proposed concept, the wireless energy monitoring system is designed to continuously monitor the electrical parameters from the digital meter and to cut the power supply through wireless techniques, if any short circuit or over load utilization (Edward, 2014).

Data can be collected at each and every second's basis. As there is no human intervention in the entire process, there is no chance of human error and corruption. In the extremely bad weather conditions like heavy snow, rain, storm, etc., the system will not hamper on collecting (Cheng et al., 2014) (Fig. 2).

- Advantages: automation has been evaluated. So, the chance of human error and corruption will be reduced

- In the extremely bad weather conditions like heavy snow, rain, storm, etc., the system will not hamper on collecting

- Illegal social activity avoided (power theft)

Internet of things: The IoT allows objects to be sensed or controlled remotely across existing network infrastructure, creating opportunities for more direct integration of the physical world into computer-based systems and resulting in improved efficiency, accuracy and economic benefit in addition to reduced human intervention. When IoT is augmented with sensors and actuators, the technology becomes an instance of the more general class of cyber-physical system which also encompasses technologies such as smart grids, virtual power plants, smart homes and smart cities. Each thing is uniquely identified through its embedded computing system but is able to interoperate within the existing internet infrastructure (Sahana et al., 2015) (Fig. 3).

Architectural model: When the various appliances of the household consume energy the energy meter reads the reading continuously and this consumed load can be seen on meter (Fig. 4). We can see that the LED on meter continuously blinks which counts the meter reading. Based on. The blinking, the units are counted. Normally, 3200 blinks is one unit. In our project, we are trying to develop, a system in which Arduino Uno act as main controller which continuously monitor energy meter (Martirano et al., 2015). As per the blinking of LED on energy meter the Arduino will measure the unit consumption. The measured reading with the calculation 
Conventional energy meter

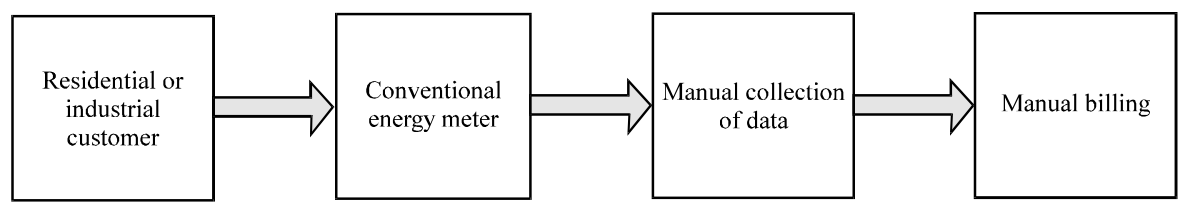

Smart meter system

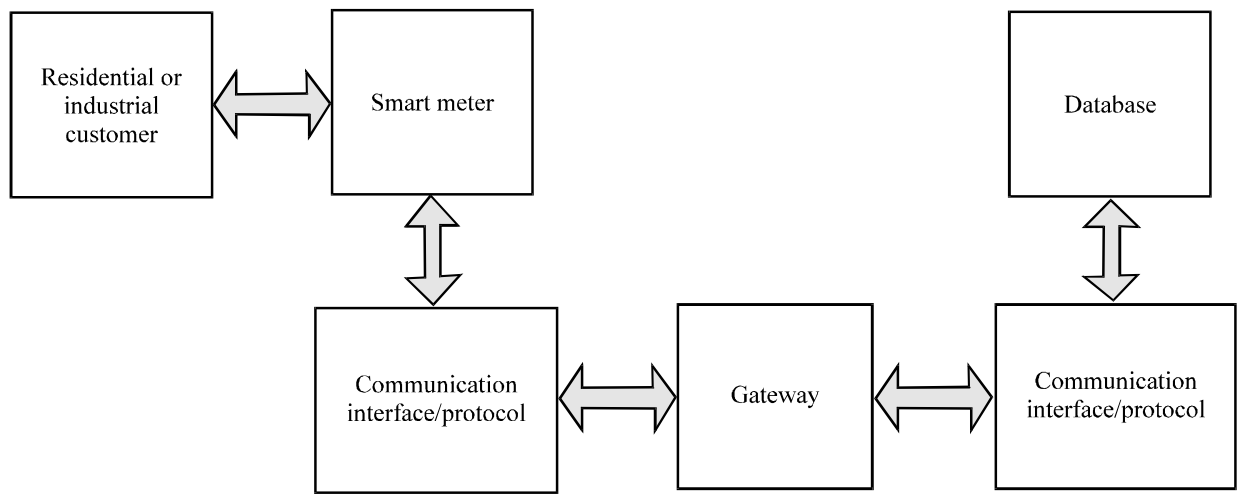

Fig. 2: Comparison of conventional and smart meter system

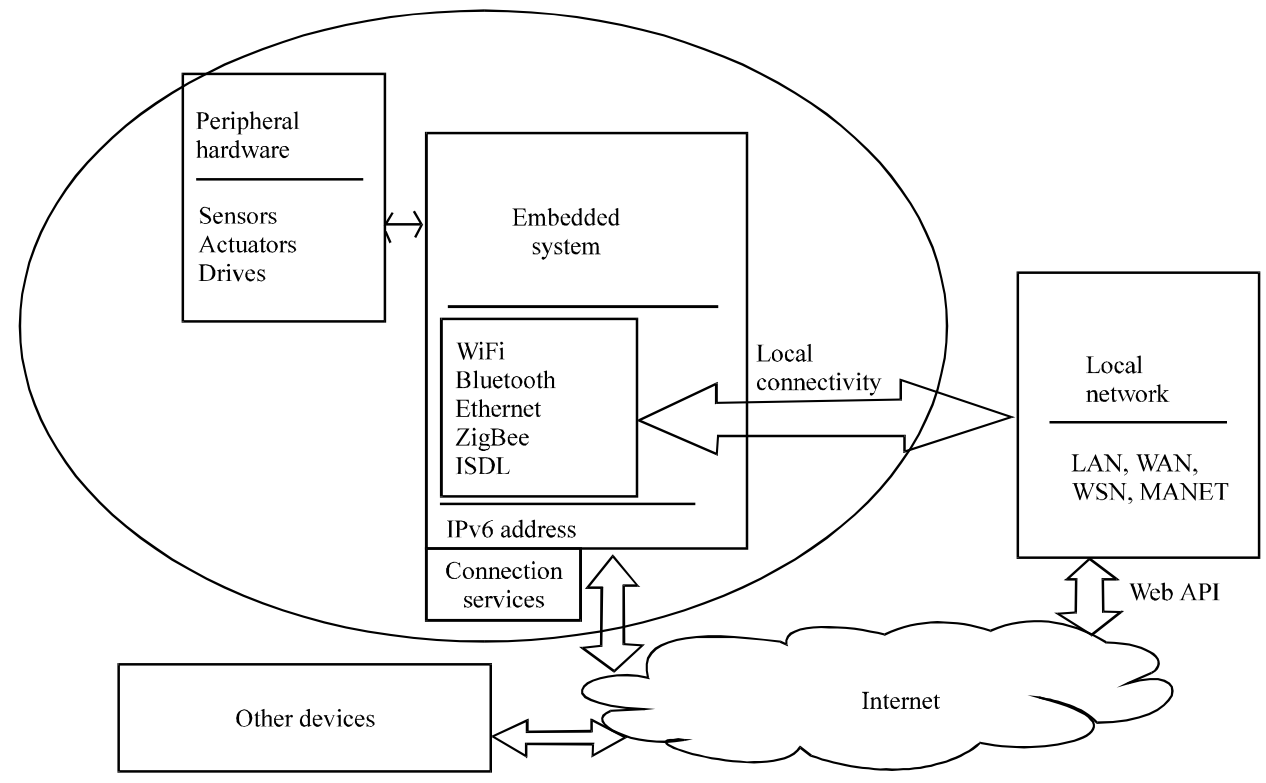

Fig. 3: IoT interfacing hardware

of the cost will be continuously displayed on web page that, we have designed. Threshold value can be set on webpage with the help of Wi-Fi as per the consumer's requirement. When the consumers reading will be near about to the set threshold value it will send a notification value to the consumer (Widmer, 2014). This threshold value notification will increase the awareness amongst the consumer about the energy. When the consumer gets the notification he can visit the webpage and change the threshold value. If the consumer is not aware with the threshold notification, then, the meter will automatically get off. Then, the consumer has to visit the webpage again and increment the threshold value. By the incrimination, the meter will automatically get on. Finally, the overall monthly bill with cost will be sent to customer as well as service provider in the form of text at 1st day of every month. 


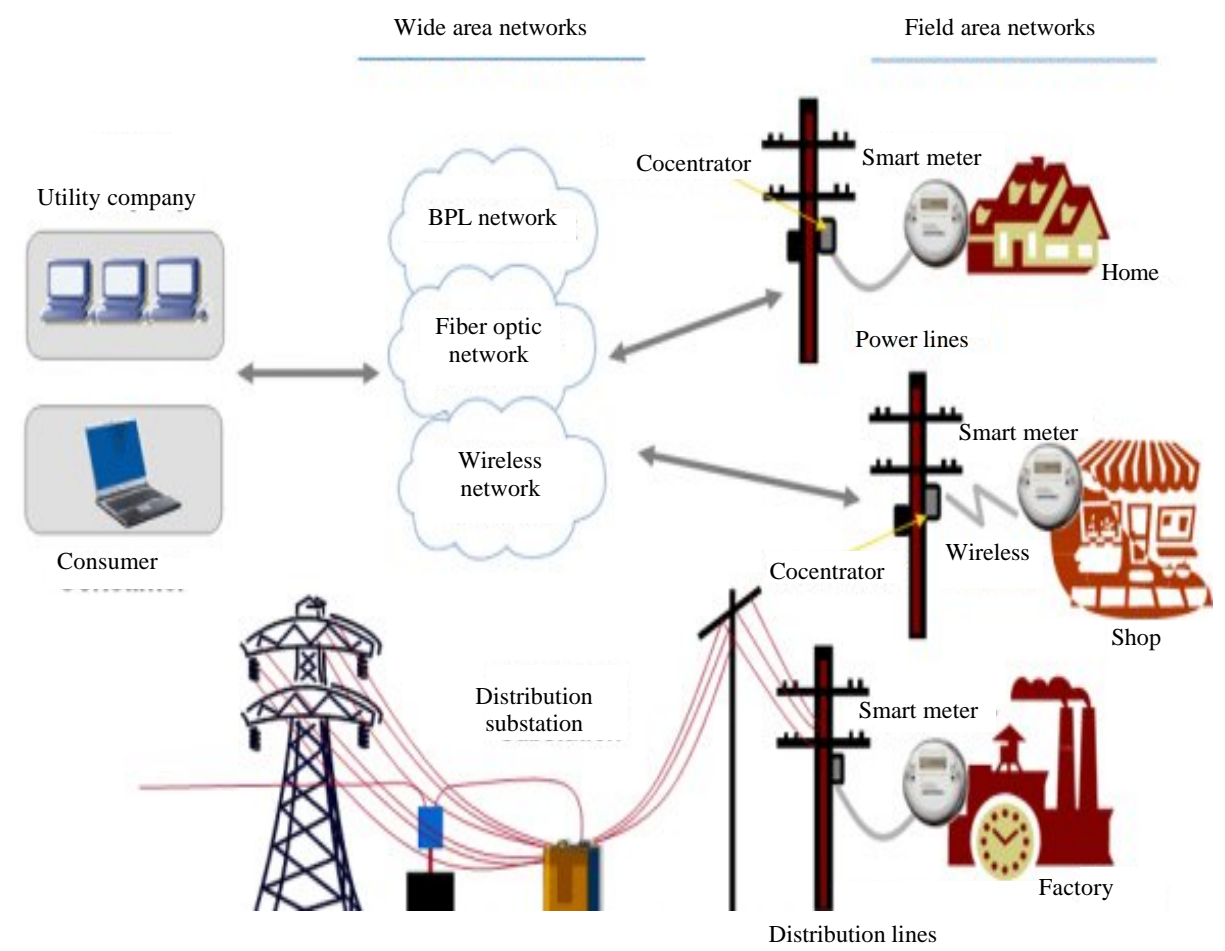

Fig. 4: Architectural model

\section{RESULTS AND DISCUSSION}

\section{Output verification and analysis Hardware description}

Arduino: Arduino Uno 328 which consists of 14 digital input/output pins, 6 pins used as PWM outputs provided with 6 analog inputs in-build with of $16 \mathrm{MHz}$ crystal oscillator, USB connection, power jack, ICSP header and a reset button. This Arduino Uno can be powered via. the USB connection or with an external power supply. The power source is selected automatically. External (non-USB) power can come either from an AC-to-DC adapter (wall-wart) or battery. The adapter can be connected by plugging (Fig. 5).

Driver circuit: A driver is an electrical circuit or other electronic component which is used to control another circuit or component such as a high-power transistor, Liquid Crystal Display (LCD). They are usually used to regulate current flowing through a circuit or to control other factors such as other components, some devices in the circuit. For example, for a specialized integrated circuit that controls high-power switches in switched-mode power converters. An amplifier can also be considered a driver for loudspeakers or a voltage regulator that keeps an attached component operating within a broad range of input voltages. Typically, the driver stages of a circuit requires different characteristics to other circuit stages, a
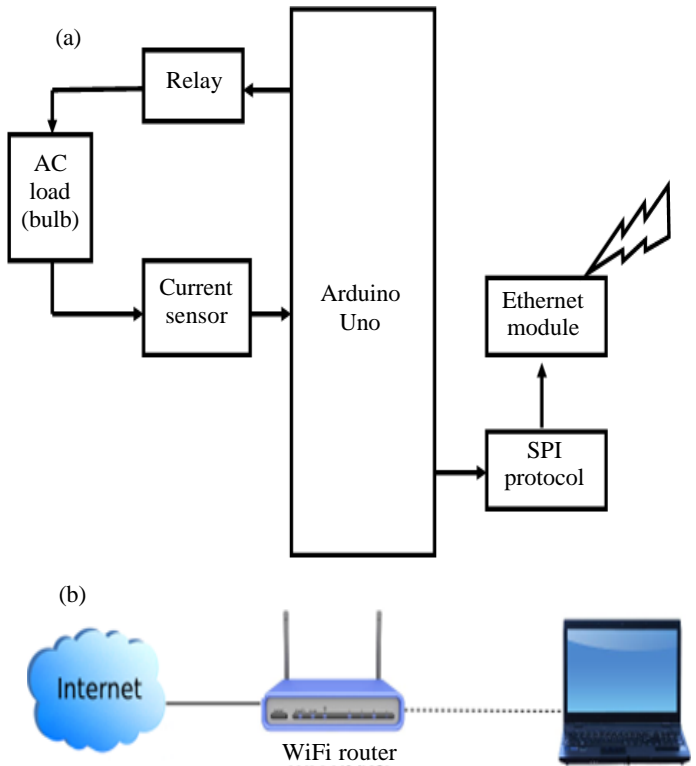

Fig. 5a, b: Block diagram of energy monitoring system using IoT

transistor power amplifier circuit which typically the driver circuit requires current gain, often the ability to discharge the following transistor bases rapidly and low output impedance to avoid or minimize distortion. 
Switch device: A network switch (also called switching hub, bridging hub, MAC bridge, it is a computer networking device that connects devices together on a computer network by using packet switching to receive or process and forward data to the destination device.

Signal condition: This signal conditioning is used for the manipulation of a signal that prepares it for the next stage of processing. Many applications involve environmental or structural measurement such as temperature and vibration, from sensors (Bhimte et al., 2015).

MAX232: MAX232 is designed by maxim integrated products. This IC is widely used in RS232 communication systems in which the conversion of voltage level is required to make TTL devices to be compatible with PC serial port and vice versa. This chip contains charge pumps which pumps the voltage to the desired level. It can be powered by a single +5 $\mathrm{V}$ power supply and its output can reach $\pm 7.5 \mathrm{~V}$. MAX232 comes in 16 Pin Dip and many other packages and it contains dual drivers. It can be used as a hardware layer convertor for 2 systems to communicate simultaneously. MAX232 is one of the versatile IC to use in most of the signal voltage level conversion problems.

WiFi module: The ESP8266 WiFi module is a self-contained SOC with integrated TCP/IP protocol stack that can give any microcontroller access to your WiFi network.

Service provider: The service provider is accessed using (SPI) Serial Peripheral Interface bus is a synchronous serial communication interface specification used for short distance communication, primarily in embedded systems. The interface was developed by Motorola and has become a de-facto standard. Typical applications include secure digital cards and liquid crystal displays. The SPI bus which operates at full duplex (means, signals carrying data can go in both directions simultaneously) is a synchronous type data link setup with a Master/Slave interface and can support up to 1 mega baud or $10 \mathrm{Mbps}$ of speed. Both single-master and multi-master protocols are possible in SPI. But the multi-master bus is rarely used and looks awkward and is usually limited to a single slave (Bhimte et al., 2015).
Web page: A document commonly written in HyperText Markup Language (HTML) that is accessible through the internet or other network using an internet browser. A web page is accessed by entering a URL address and may contain text, graphics and hyperlinks to other web pages and files (Arasteh et al., 2016).

Consumer: The person who pays the billing amount which is been by the energy meter through online. This hardware description explains that this reduces the manual task.

Software description (Arduino IDE): The Arduino Integrated Development Environment or Arduino Software (IDE) includes a text editor for writing code, a message area, a text console, a toolbar with buttons for common functions and a series of menus. It connects to the Arduino and Genuino hardware to upload programs and communicate with them (Fig. 6).

Writing sketches: Programs written using Arduino Software (IDE) are called sketches. These sketches are written in the text editor and are saved with the file extension ino. The editor has features for cutting/pasting and for searching/replacing text. The message area gives feedback while saving and exporting and also displays errors. The console displays text output by the Arduino Software (IDE) including complete error messages and other information. The bottom righthand corner of the window displays the configured board and serial port. The toolbar buttons allow you to verify and upload programs, create, open and save sketches and open the serial monitor (Fig. 7 and 8).

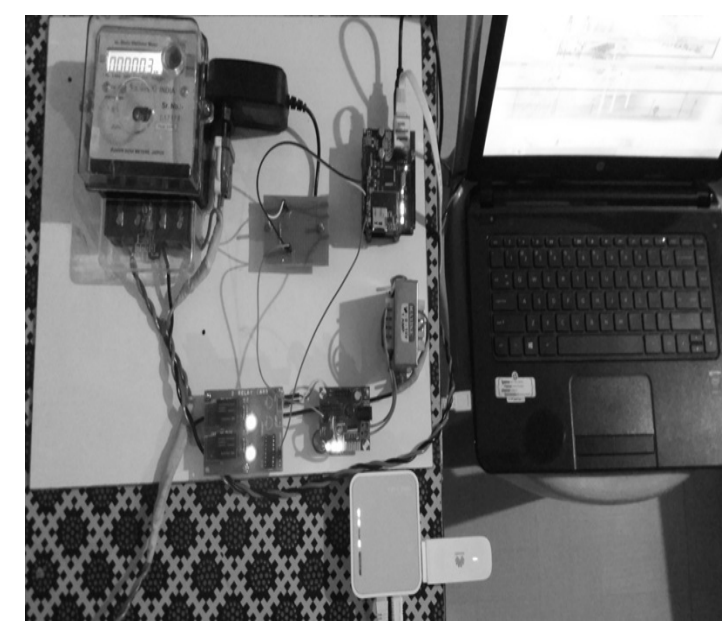

Fig. 6: Monitoring hardware interface 


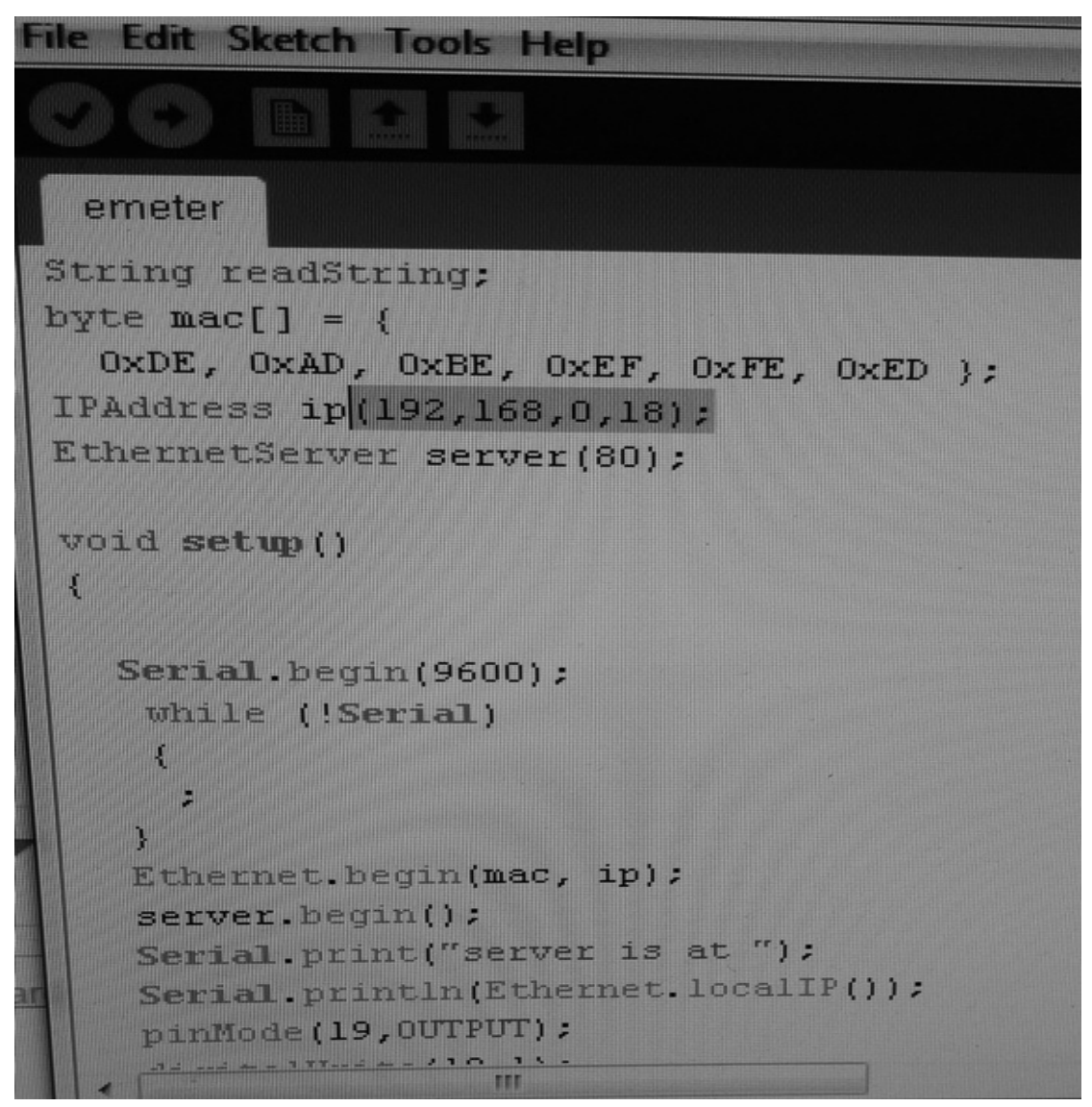

Fig. 7: Software coding

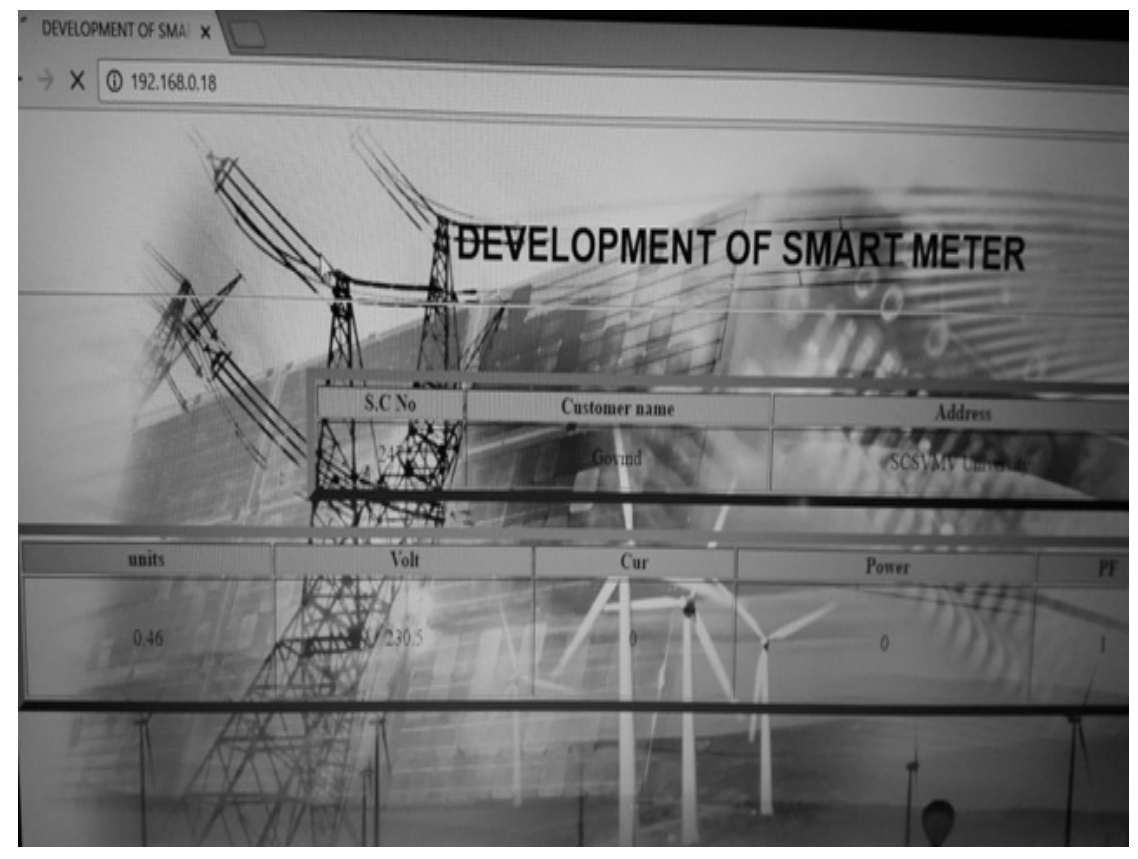

Fig. 8: Web interface for monitoring power consumption 


\section{CONCLUSION}

An IoT based low cost smart energy monitoring system designed with both hardware and software tool. The proposed model is used to simulate and analyse the energy consumption in residential and industrial areas and also the energy unit reading is measured. The system reduces the wastage of energy and it is highly accurate and compatible, hence, the system implemented with low cost and advanced techniques.

\section{RECOMMENDATION}

In future, we planned to implement the energy monitoring system with cloud techniques to get the huge the process in the smart grid.

\section{REFERENCES}

Arasteh, H., V. Hosseinnezhad, V. Loia, A. Tommasetti and O. Troisi et al., 2016. Iot-based smart cities: A survey. Proceeding of the 2016 IEEE 16th International Conference on Environment and Electrical Engineering (EEEIC), June 7-10, 2016, IEEE, Florence, Italy, ISBN:978-1-5090-2321-9, pp: 1-6.

Bhimte, A., R.K. Mathew and S. Kumaravel, 2015. Development of smart energy meter in LabVIEW for power distribution systems. Proceedings of the 2015 Annual IEEE India Conference (INDICON), December 17-20, 2015, IEEE, New Delhi, India, ISBN:978-1-4673-7398-2, pp: 1-6.

Cheng, Y., H. Yang, J. Xiao and X. Hou, 2014. Running state evaluation of electric energy meter. Proceedings of the 2014 IEEE Workshop on Electronics, Computer and Applications, May 8-9, 2014, IEEE, Ottawa, Canada, ISBN:978-1-4799-4566-5, pp: 43-46.

Das, H. and L.C. Saikia, 2015. GSM enabled smart energy meter and automation of home appliances. Proceedings of the 2015 International Conference on Energy, Power and Environment: Towards Sustainable Growth (ICEPE), June 12-13, 2015, IEEE, Shillong, India, ISBN:978-1-4673-6504-8, pp: 1-5.

De Capua, C., G. Lipari, M. Lugara and R. Morello, 2014. A smart energy meter for power grids. Proceedings of the 2014 IEEE International Conference on Instrumentation and Measurement Technology (I2MTC), May 12-15, 2014, IEEE, Montevideo, Uruguay, ISBN:978-1-4673-6386-0, pp: 878-883.
Edward, O.O., 2014. An energy meter reader with load control capacity and secure switching using a password based relay circuit. Proceedings of the 2014 Annual Global Online Conference on Information and Computer Technology (GOCICT), December 3-5, 2014, IEEE, Louisville, Kentucky, USA., ISBN:978-1-4799-8311-7, pp: 58-63.

Martirano, L., M. Manganelli and D. Sbordone, 2015. Design and classification of smart metering systems for the energy diagnosis of buildings. Proceedings of the 2015 IEEE International Conference on Smart Energy Grid Engineering (SEGE), Auguest 17-19, 2015,IEEE, Oshawa, Canada, ISBN:978-1-4673-7932-8, pp: $1-7$.

Molderink, A., V. Bakker, M.G. Bosman, J.L. Hurink and G.J. Smit, 2010. Management and control of domestic smart grid technology. IEEE. Trans. Smart Grid, 1: 109-119.

Ruzzelli, A.G., C. Nicolas, A. Schoofs and G.M. O'Hare, 2010. Real-time recognition and profiling of appliances through a single electricity sensor. Proceedings of the 2010 7th Annual IEEE Communications Society Conference on Sensor Mesh and Ad Hoc Communications and Networks (SECON), June 21-25, 2010, IEEE, Boston, Massachusetts, USA., ISBN:978-1-4244-7150-8, pp: $1-9$.

Sahana, M.N., S. Anjana, S. Ankith, K. Natarajan and K.R. Shobha et al., 2015. Home energy management leveraging open IoT protocol stack. Proceedning of the 2015 IEEE International Conforence on Recent Advances in Intelligent Computational Systems (RAICS), December 10-12, 2015, IEEE, Trivandrum, India, ISBN:978-1-4673-6669-4, pp: 370-375.

Tariq, T., 2014. Smart energy management in a smart environment using a mobile device. Proceedings of the 2014 Global Summit on Computer and Information Technology (GSCIT), June 14-16, 2014, IEEE, Sousse, Tunisia, ISBN:978-1-4799-5626-5, pp: 1-5.

Widmer, J., 2014. Billing metering using sampled values according IEC 61850-9-2 for substations. Proceedings of the 2014 International Conference on Saudi Arabia Smart Grid Conference (SASG), December 14-17, 2014 , IEEE, Jeddah, Saudi Arabia, ISBN:978-1-4799-6157-3, pp: $1-7$. 